\title{
A Novel Nitric Oxide Donor, S-Nitroso-N- Pivaloyl-D-Penicillamine, Activates a Non- Neuronal Cardiac Cholinergic System to Synthesize Acetylcholine and Augments Cardiac Function
}

\author{
Shino Oikawa ${ }^{a}$ Yuko Kaia Asuka Mano ${ }^{a}$ Shigeo Nakamurab \\ Yoshihiko Kakinuma ${ }^{a}$ \\ aDepartment of Bioregulatory Science (Physiology), Nippon Medical School Graduate School of \\ Medicine, Tokyo, Japan, 'Department of Chemistry, Nippon Medical School, Tokyo, Japan
}

\section{Key Words}

S-nitroso-N-pivaloyl-D-penicillamine (SNPiP) - Non-neuronal cardiac cholinergic system $(\mathrm{NNCCS}) \cdot$ Acetylcholine $\bullet$ Heart $・$ Nitric oxide (NO)

\begin{abstract}
Background/Aims: In a previous study, we reported that cardiomyocytes were equipped with non-neuronal cardiac cholinergic system (NNCCS) to synthesize acetylcholine (ACh), which is indispensable for maintaining the basic physiological cardiac functions. The aim of this study was to identify and characterize a pharmacological inducer of NNCCS. Methods: To identify a pharmacological inducer of NNCCS, we screened several chemical compounds with chemical structures similar to the structure of S-nitroso- $\mathrm{N}$-acetyl-DL-penicillamine (SNAP). Preliminary investigation revealed that SNAP is an inducer of non-neuronal ACh synthesis. We screened potential pharmacological inducers in H9c2 and HEK293 cells using western blot analysis, luciferase assay, and measurements of intracellular CGMP, NO 2 and ACh levels. The effects of the screened compound on cardiac function of male C57BL6 mice were also evaluated using cardiac catheter system. Results: Among the tested compounds, we selected S-nitroso- $\mathrm{N}$ pivaloyl-D-penicillamine (SNPiP), which gradually elevated the intracellular cGMP levels and nitric oxide (NO) levels in H9c2 and HEK293 cells. These elevated levels resulted in the gradual transactivation and translation of the choline acetyltransferase gene. Additionally, in vitro and in vivo SNPiP treatment elevated ACh levels for $72 \mathrm{~h}$. SNPiP-treated mice upregulated their cardiac function without tachycardia but with enhanced diastolic function resulting in improved cardiac output. The effect of SNPiP was dependent on SNPiP nitroso group as
\end{abstract}

S. Oikawa and Y. Kai contributed equally to this work.

\footnotetext{
Yoshihiko Kakinuma, M.D., Ph.D. Dept. of Bioregulatory Science (Physiology), Nippon Medical School Graduate School of Medicine 


\section{Cellular Physiology Cell Physiol Biochem 2019;52;922-934 \\ \begin{tabular}{ll|l} 
DOI: 10.33594/000000064 & (c) 2019 The Author(s). Published by \\
Cell Physiol Biochem Press GmbH\&Co. KG
\end{tabular} \\ Oikawa et al.: S-Nitroso-N-Pivaloyl-D-Penicillamine Activates Cardiac ACh Synthesis}

verified by the ineffectiveness of $\mathrm{N}$-pivaloyl-D-penicillamine (PiP), which lacks the nitroso group. Conclusion: SNPiP is identified to be one of the important pharmacological candidates for induction of NNCCS.

(c) 2019 The Author(s). Published by Cell Physiol Biochem Press GmbH\&Co. KG

\section{Introduction}

We have thus far advocated a novel concept that cardiomyocytes possess a system to produce acetylcholine (ACh), a non-neuronal cardiac cholinergic system (NNCCS). It is crucial for maintaining the indispensable physiological functions in the heart as well as the brain, which includes conferring ischemia-resistance, accelerated cell-cell communication and vagus nerve-mediated anti-stress effects on the central nervous system (CNS) [1-4]. We have also explored a modality to eventually activate or accelerate NNCCS, and thereafter, we reported that NNCCS can be activated pharmacologically and physically $[1,5]$. We have previously demonstrated that donepezil, an acetylcholinesterase inhibitor, is an inducer for choline acetyltransferase (ChAT). Donepezil transcriptionally and translationally upregulates the enzyme to consequently enhance the ACh production in cultured cardiomyocytes [1]. Moreover, we have also reported that remote ischemic preconditioning can also upregulate NNCCS $[2,5]$. This modality, with its capability to induce NNCCS, led us to hypothesize that NNCCS may be regulated through the CNS pathway via the vagus nerve that directly connects the heart with the brain [4-7].

Furthermore, to identify agents that can directly upregulate NNCCS, which is independent of the effects related to neuronal activity and synaptic modulation in the cholinergic system, we screened for several pharmacological agents. Among these, we initially focused on compounds with chemical structures that resembled the structure of a nitric oxide (NO) donor, S-nitroso- $N$-acetyl-DL-penicillamine (SNAP). Additionally, we synthesized compounds possessing different functional groups based on the SNAP structure to investigate its property to upregulate NNCCS. Consequently, we identified a compound, SNPiP, that could enhance NNCCS to produce cardiac ACh, leading to increased cardiac function.

\section{Materials and Methods}

Synthesis of a new compound possessing a backbone that partly resembles the chemical structure of SNAP SNAP is a chemical compound comprising $S$-nitrosothiol and penicillamine and has gained attention due to its NO releasing property [8]. Our preliminary experiments revealed that SNAP-treated cells upregulated the ChAT protein expression and ACh synthesis. Therefore, we used a compound possessing the basic chemical backbone of SNAP and further modified their functional groups attached to the base structure. In the penicillamine structure, we specifically focused on the amino group and synthesized several compounds resembling the basic SNAP structure with this group. Various other structures were attached to the amino group, including the reported reference compounds. We prepared $S$-nitroso- $N$-benzoyl-D-penicillamine (SNBP), $S$-nitroso- $N$-succinoyl-D-penicillamine (SNSP), $S$-nitroso- $N$-pivaloyl-D-penicillamine (SNPiP) as well as the previously reported compounds $S$-nitroso- $N$-heptanoyl-D-penicillamine (SNHP) and $S$-nitroso$N$-valeryl-D-penicillamine (SNVP). SNPiP was synthesized in a $20 \mathrm{~mL}$ tetrahydrofuran: water (4:1) solution. The reaction was carried out at $0{ }^{\circ} \mathrm{C}$ for $30 \mathrm{~min} .6 .7 \mathrm{mmol}(1.0 \mathrm{~g})$ of D-penicillamine was reacted with 6.7 mmol $(0.81 \mathrm{~g})$ of pivaloyl chloride in the presence of triethylamine $(13.4 \mathrm{mmol})$. The reaction was allowed to continue for further $12 \mathrm{~h}$ at room temperature. Tetrahydrofuran was evaporated, and this mixture was added to hydrochloric acid to obtain $621 \mathrm{mg}$ of $\mathrm{N}$-pivaloyl-D-penicillamine (PiP). The reaction between $2.5 \mathrm{mmol}$ PiP with $5 \mathrm{mmol}$ sodium nitrate resulted in $237 \mathrm{mg}$ of SNPiP, a green powder. NMR analysis of the compound confirmed its high purity. The other compounds were also synthesized via the modified methods used for SNPiP synthesis. The chemical structure of SNPiP is shown in Fig. 1 in comparison with SNAP and PiP. SNPiP has structural similarity to PiP; however, PiP lacked the nitroso group. PiP was used 


\section{Cellular Physiology Cell Physiol Biochem 2019;52;922-934

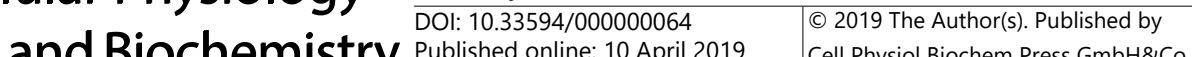 \\ Published online: 10 April 2019 Cell Physiol Biochem Press GmbH\&Co. KG \\ Oikawa et al.: S-Nitroso-N-Pivaloyl-D-Penicillamine Activates Cardiac ACh Synthesis}

to understand the role of nitroso group in the beneficial effects of SNPiP. In a preliminary experiment, HEK293 cells were confirmed to possess the ability to synthesize ACh, which can be easily detected by high performance liquid chromatography (HPLC).

Luciferase assay for measuring ChAT transcriptional activity

As previously reported in our study, ChAT promoter region of $2.0 \mathrm{~kb}$ length was subcloned into a luciferase vector (Toyo B-Net Co., LTD, Tokyo, Japan) [1]. HEK293 cells were cultured on type I collagen (Nitta Gelatin Inc., Osaka, Japan)-coated dish in low glucose Dulbecco's Modified Eagle Medium (DMEM; FUJIFILM Wako Pure Chemical Corporation, Osaka Japan) with $10 \%$ fetal bovine serum (FBS; Thermo Fisher Scientific K.K., Tokyo, Japan) and antibiotics (FUJIFILM Wako Pure Chemical Corporation). HEK 293 cells or H9c2 cells (1x10 cells / well) cultured in a 24-well plate were transfected using Effectene Transfection Reagent (QIAGEN, Venlo, Netherlands) according to the manufacturer's instructions. Cells were treated with either one of the synthesized chemical compounds or phosphate buffered saline (PBS) $48 \mathrm{~h}$ post-transfection. Cells were collected at 8, 16, or $24 \mathrm{~h}$ after the treatment and lysed to obtain samples for measuring the luciferase activity. The concentration of SNPiP (dissolved in dimethyl sulfoxide) for in vitro experiments was optimized at $1 \mu \mathrm{M}$. Data were obtained from six to nine independent experiments.

\section{Western blot analysis}

As previously reported, HEK 293 cells, H9c2 cells or C6 cells, treated with compounds, were lysed in a sampling buffer [1-4]. Proteins were isolated from the excised liver, heart or skeletal muscle of C57BL6 mouse. Equal amounts of protein from different treatment groups were electrophoresed in $10 \%$ acrylamide gel and transferred onto a PVDF membrane. The membrane was blocked with 4\% skim milk and incubated with a goat anti-ChAT polyclonal antibody (1:2000, Merck Millipore, Temecula, CA, USA), followed by incubation with an anti-goat IgG antibody (1:1000, Santa Cruz Biotechnology, CA, USA) at room temperature. The signals were detected by D-DiGit Gel Scanner (LI-COR Biotechnology, Lincoln, NE, USA) via ImmunoStar LD (FUJIFILM Wako Pure Chemical Corporation). Representative data were obtained from four to six independent experiments.

\section{Measurement of intracellular ACh contents}

Intracellular ACh contents were measured via HPLC following a previously described method [1]. HEK293 cells cultured in a $10 \mathrm{~cm}$ culture dish were lysed with $1 \mathrm{~mL}$ lysis buffer comprising $0.1 \mathrm{M}$ perchloric acid, $0.1 \mathrm{mM}$ EDTA, $10^{-8} \mathrm{M}$ isopropyl homocholine and $0.1 \mathrm{mM}$ physostigmine. The samples were centrifuged for $15 \mathrm{~min}$ at $0^{\circ} \mathrm{C}$ with $20,000 \mathrm{x} \mathrm{g}$, and the supernatant was adjusted to a $\mathrm{pH}>6.5$ with $1 \mathrm{M} \mathrm{KHCO}_{3}$ and was filtrated through a Millipore column (Amicon Ultra $0.5 \mathrm{~mL}$ ). Ten microliters of the sample was used for measuring ACh levels. HEK293 cells treated with the compound of interest were lysed $24 \mathrm{~h}$ to $72 \mathrm{~h}$ posttreatment for measuring the ACh levels. For in vivo measurements, the murine cardiac ventricles alone were excised. Thereafter, the procedure to measure ACh levels was identical to those mentioned above $(n=3-6$ for each time point). 


\section{Cellular Physiology Cell Physiol Biochem 2019;52;922-934 \\ \begin{tabular}{ll|l} 
and Biochemistry & $\begin{array}{l}\text { DOI: } 10.33594 / 000000064 \\
\text { Published online: } 10 \text { April } 2019\end{array}$ & ( 2019 The Author(s). Published by \\
Cell Physiol Biochem Press GmbH\&Co. KG
\end{tabular}

cGMP measurement of HEK293 cells

cGMP was measured in compound-treated HEK293 cells using the Cyclic GMP EIA kit (Cayman Chemical Company, Ann Arbor, MI, USA) as per manufacturer's instructions, and the absorbance was measured at a wavelength of $420 \mathrm{~nm}$. Sampling of the compound-treated HEK293 cells was performed within 10 min of the treatment $(n=2-4$ for each time point).

\section{NO measurement}

HEK293 cells cultured in a $10 \mathrm{~cm}$ culture dish, treated with SNPiP, were scraped in PBS. The cells were sonicated at level 12 for 15 s using Microson Ultrasonic Cell Disruptor XL2000 (Farmingdale, NY, USA), followed by centrifugation at $14,000 \mathrm{rpm}$ at $4^{\circ} \mathrm{C}$ for $10 \mathrm{~min}$. The supernatant was used for $\mathrm{NO}_{2}$ measurement using the QuantiChrom Nitric Oxide Assay Kit (BioAssay Systems, Heyward, CA, USA). NO was oxidized to $\mathrm{NO}_{2}$ and hence the level of $\mathrm{NO}_{2}$ is a measure of NO production in the cell. The sample optical density (OD) was measured at $540 \mathrm{~nm}$ and cellular $\mathrm{NO}_{2}$ levels, and hence the NO production, were calculated from the standard curve ( $\mathrm{n}=2-4$ for each time point).

\section{Evaluation of cardiac function in compound-treated mice}

The murine cardiac function was measured using a catheter system (ADVantage system, Transonic Science, Inc., Ithaca, NY, USA) [4]. Male C57BL6 mice ( $\mathrm{n}=8-15$ for each group) were intraperitoneally injected with SNPiP or PiP (60 pmol/g BW/dose) once. The cardiac function was measured $72 \mathrm{~h}$ later. The dose was optimized based on the hemodynamic adverse effects observed in our preliminary experiments [3].

\section{Statistical analysis}

Data were represented as means \pm standard errors. Comparison between two groups was performed using an unpaired Student's $t$-test. For multiple group comparisons, difference was evaluated by nonrepeated one-way ANOVA, followed by post-hoc Dunnett's tests. Differences were considered significant at a $P$ value $<0.05$.

\section{Results}

SNAP increases cellular ChAT protein expression

H9c2 cells derived from rat myocardium were treated with SNAP at various concentrations $(1 \mathrm{nM}-1000 \mu \mathrm{M})$ to induce ChAT protein expression. The ChAT protein expression increased $8 \mathrm{~h}$ after treatment with $1 \mu \mathrm{M}$ SNAP treatment $[148 \pm 2 \%, P<0.01$, $\mathrm{F}(2,15)=60.4]$ and peaked at $16 \mathrm{~h}[183 \pm 3 \%, P<0.01, \mathrm{~F}(2,15)=208.7]$ (Fig. 2A).

Moreover, SNAP increased the ChAT expression not only in H9c2 cells but also in HEK293 cells, and elevated ChAT expression at $16 \mathrm{~h}$ [lane 6 in Fig. 2B, $141 \pm 3 \%, P<0.01, \mathrm{~F}(6,21)$ $=133.4]$. SNAP showed similar results in rat C6 cells (data not shown). Collectively, this suggested that SNAP activated the pivotal ACh synthesizing enzyme in various types of cells.

Transcriptional activity is increased by SNAP-derivatives with a different time course

To investigate whether the chemical compounds with structure similar to that of SNAP transactivated the ChAT promoter activity, we compared the luciferase activity of compoundtreated (1 $\mu \mathrm{M}$ each) HEK293 cells. As shown in Fig. 2B, luciferase activity at $8 \mathrm{~h}$ after the treatment was slightly higher in SNHP- (lane 2: $113.8 \pm 4.9 \%$ ), SNVP- (lane 4: $118.5 \pm 1.6$ $\%$ ), and SNPiP- (lane 5: $110.0 \pm 4.6 \%$ ) treated cells compared to the luciferase activity in SNAP-treated cells (lane 6: $105.9 \pm 0.8 \%$ ). Furthermore, in contrast to the other derivatives, a delayed elevation in ChAT protein expression was observed in SNPiP-treated cells at $16 \mathrm{~h}$ [Fig. 2B, lane 5: $158 \pm 2 \%, P<0.01$ vs. control and SNAP, F(6, 21) $=133.4$ ], suggesting that SNPiP has a distinctive pattern for ChAT upregulation. Similarly, a delayed elevation in ChAT protein expression was observed at $48 \mathrm{~h}$ (Fig. $2 \mathrm{C}, 132 \pm 3 \%$ vs control, $P<0.01, t=20.87$ ), suggesting that the ChAT expression was gradually activated in SNPiP-treated cells. 


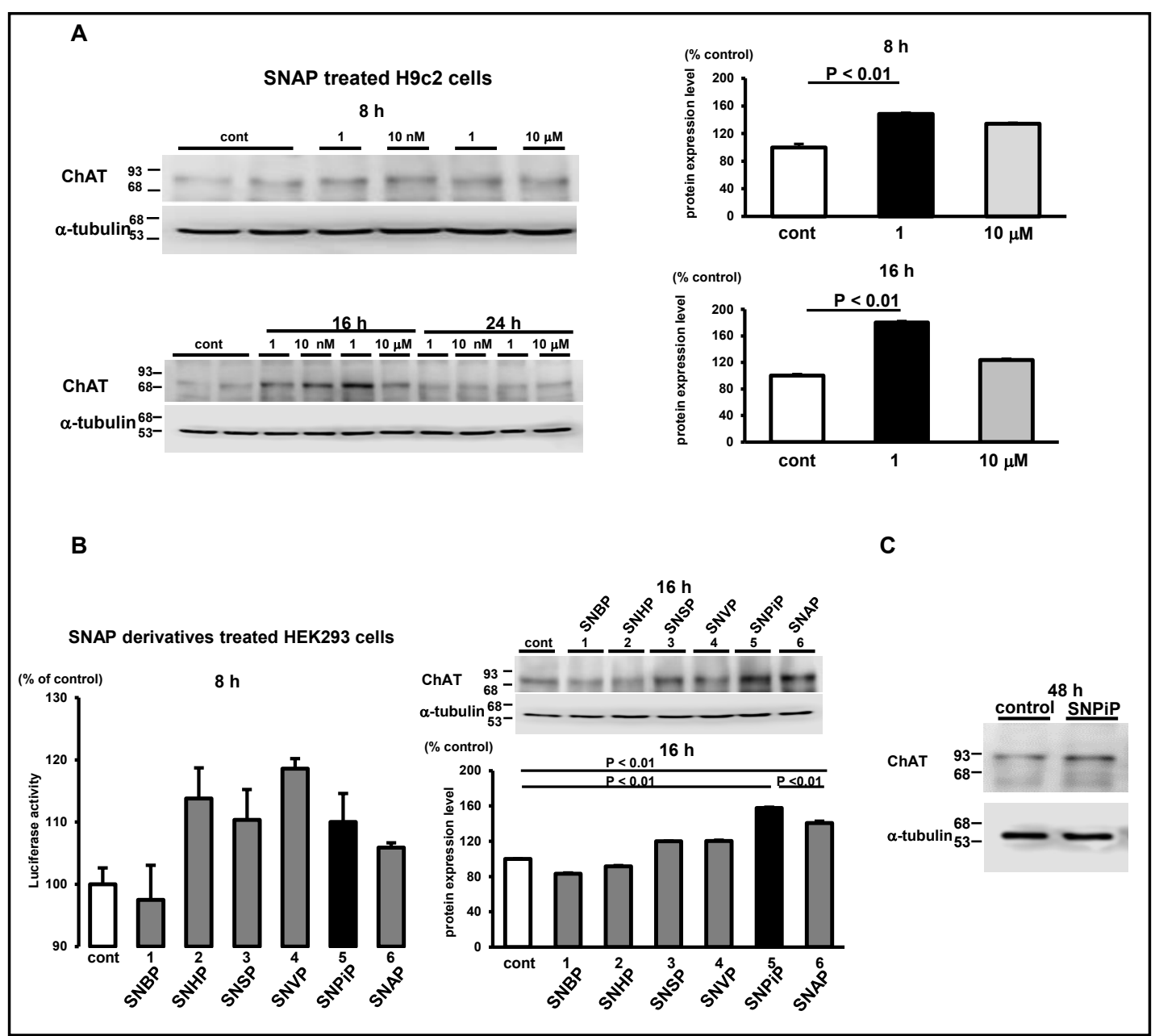

Fig. 2. SNPiP increases choline acetyltransferase (ChAT) expression not only in H9c2 cells but also in HEK293 cells. A. ChAT protein expression in $1 \mathrm{M} \mathrm{SNPiP-treated} \mathrm{H9c2} \mathrm{cells} \mathrm{is} \mathrm{upregulated} \mathrm{within} 8 \mathrm{~h}(\mathrm{P}<0.01)$ and attain a peak value at $16 \mathrm{~h}(\mathrm{P}<0.01)$, after which, it attenuated to the basal level at $24 \mathrm{~h}$. B. HEK293 cells respond to SNPiP treatment with upregulated ChAT protein expression at $16 \mathrm{~h}(\mathrm{P}<0.01)$ and an enhanced luciferase activity of ChAT promoter region at $8 \mathrm{~h}$. C. SNPiP continue to upregulate ChAT protein expression at $48 \mathrm{~h}$.

Intracellular ACh synthesis is upregulated by SNPiP

SNPiP enhanced the ACh levels at 16 h (Fig. 3, lane 5: $6.6 \pm 0.2 \times 10^{-9} \mathrm{M}$ ) in HEK293 cells. The other compounds were also able to moderately increase the ACh levels (lane 1-4, 6). These results suggested that SNPiP activated ACh synthesis gradually. Therefore, further experiments focused on SNPiP.

\section{SNPiP upregulates cellular NO production at a slower rate than SNAP}

As shown in Fig. 4, the NO release by SNAP rapidly increased $\left[\mathrm{NO}_{2}: 1.47 \pm 0.4 \mathrm{nmol}\right.$ (20 min) vs. $0.98 \pm 0.23 \mathrm{nmol}(0 \mathrm{~min})]$, compared to the NO release of SNPiP, at $20 \mathrm{~min}$. In contrast, slow release of NO by SNPiP was observed and the release peaked at around $60 \mathrm{~min}$ $\left[\mathrm{NO}_{2}: 2.26 \pm 0.62 \mathrm{nmol}(60 \mathrm{~min})\right.$ vs. $\left.0.97 \pm 0.03 \mathrm{nmol}(0 \mathrm{~min})\right]$. This suggested that in SNPiP the NO release was gradual and the peak release was delayed. 
SNPiP modulates cGMPlevels more slowly than SNAP

Next, we investigated the time course of cGMP levels in the two treated cells (Fig. 5). Concurring with the results from the NO release, SNAP rapidly upregulated the cGMP levels, which peaked within $2 \mathrm{~min}$ of treatment $0.58 \pm 0.02$ pmol/ $\mathrm{mL})$. In contrast, SNPiP gradually upregulated the cGMP levels, which did not attain a peak value during the observation period of $10 \mathrm{~min}$ (10 min: $0.68 \pm 0.22 \mathrm{pmol} / \mathrm{mL}$; Fig. 5). Even when compared with the peak levels obtained with SNBP (at $1 \mathrm{~min}$ ), SNPiP altered cGMP response in a unique pattern, suggesting that SNPiP slowly modulates a signal transduction messenger, compared to the cGMP response by SNAP-treatment.

SNPiP activates ACh synthesis with its delayed mode

Based on the results thus far, it was expected that SNPiP enhanced ACh synthesis at a slower rate than SNAP. Further, HEK293 cells were treated with $1 \mu \mathrm{M}$ SNPiP and the intracellular ACh levels were measured for $72 \mathrm{~h}$ (Fig. 6). SNAP initially enhanced ACh synthesis at $8 \mathrm{~h}$, followed by a moderate increase at $36 \mathrm{~h}$, with the extents of increase being $153 \pm 6 \%$. In contrast, SNPiP continued to further enhance ACh synthesis to $264 \pm$ $5 \%$ at $48 \mathrm{~h}$ [vs. SNAP $115 \pm 1 \%, P<0.01, \mathrm{~F}(8,35)=$ 52.7] and $166.9 \pm 9 \%$ at $72 \mathrm{~h}$ [vs. SNAP $104 \pm 8 \%, P$ $<0.01, F(8,35)=52.7]$. These results clearly indicated that SNPiP upregulated ACh synthesis for a prolonged period.

SNPiP upregulates ChAT expression in the heart, leading to elevated cardiac ACh synthesis

Male C57BL6 mice were intraperitoneally injected with SNPiP, and 24-72 h later, ChAT protein expression was evaluated in the heart. At $48 \mathrm{~h}$, the cardiac ChAT protein expression was enhanced (Fig. 7), as well as at $72 \mathrm{~h}$ (Fig. 8). In contrast, upregulation of the ChAT expression was not observed in other organs such as the skeletal muscle and liver (data not shown).

ACh levels were measured in the ventricles excised from SNPiP-injected (60 pmol/g BW/dose) C57BL6 murine heart. Fig. 7 shows that at $72 \mathrm{~h}$ following the injection, the cardiac ACh level was significantly increased to $63.1 \pm 11.5 \times 10^{-8} \mathrm{M} / \mathrm{g}$ tissue, compared with the control ACh level of $18.4 \pm 4.1 \times 10^{-8} \mathrm{M} / \mathrm{g}$ tissue $(P<$ $0.01, t=3.68)$. While this upregulation was moderate at $24 \mathrm{~h}\left(45.4 \pm 20.6 \times 10^{-8} \mathrm{M} / \mathrm{g}\right.$ tissue $)$ compared to

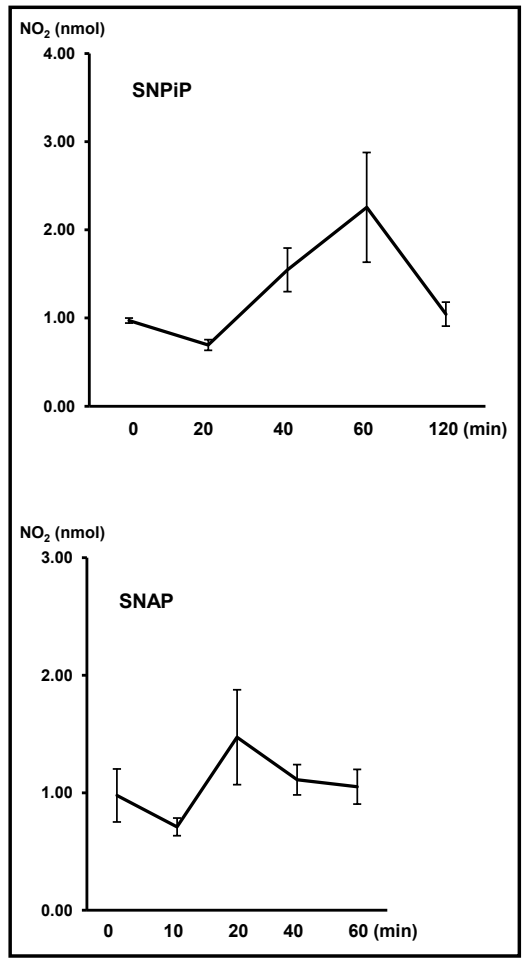

Fig. 4. Elevation of intracellular $\mathrm{NO}_{2}$ levels is delayed with SNPiP treatment compared to the $\mathrm{NO}_{2}$ levels with SNAP treatment. One micromolar of SNAP treatment rapidly increases $\mathrm{NO}_{2}$ levels in HEK293 cells within 20 min. However, the mode of SNPiP induction is in contrast to that of SNAP with the peak value shifting to $60 \mathrm{~min}$ after treatment. 
ACh levels in the control $(17.0$ $\pm 6.8 \times 10^{-8} \mathrm{M} / \mathrm{g}$ tissue, $P<0.05$, $t=2.64)$, the increase was more remarkable at $72 \mathrm{~h}$. These results suggest that SNPiP activates in vivo ACh synthesis specifically in the murine heart via NNCCS.

SNPiP enhances cardiac function in mice

Following the intraperitoneal injection of SNPiP, the cardiac function was evaluated at 24,48 , and $72 \mathrm{~h}$. While cardiac function was affected only slightly at $24 \mathrm{~h}$, a significant improvement was evident at $72 \mathrm{~h}$ (Fig. 9). At this point, stroke volume (SV) was significantly increased $(28.5 \pm$ $1.6 \mu \mathrm{L}$ vs. $20.7 \pm 1.2 \mu \mathrm{L}, P<0.01$, $t=3.67$ ) along with the enddiastolic volume (EDV: $35.3 \pm 9.1$ $\mu \mathrm{L}$ vs. $26.1 \pm 1.3 \mu \mathrm{L}, P<0.05, t=$ 2.61). Consequently, this led to an increased cardiac output (CO: $11272 \pm 901 \mu \mathrm{L} / \mathrm{min}$ vs. $8241 \pm$ $526 \mu \mathrm{L} / \mathrm{min}, P<0.01, t=2.76$ ) and increased ejection fraction (EF: $87.5 \pm 1.3 \%$ vs. $79.5 \pm 3.5 \%$, $P<0.05, t=2.17$ ) associated with increased end-systolic pressure (ESP: $91.8 \pm 2.3 \mathrm{mmHg}$ vs. 84.4 $\pm 2.7 \mathrm{mmHg}, P<0.05, t=2.04$ ). However, the heart rate (HR) was not affected ( $394 \pm 13$ vs. $399 \pm 14$, Fig. 9). Even at $24 \mathrm{~h}$, the beneficial effect of SNPiP was already visible in several parameters (SV: $35.0 \pm$ $3.1 \mu \mathrm{L}$ vs. $25.3 \pm 1.6 \mu \mathrm{L}, P<0.01, t$ $=2.79$; EDV: $40.7 \pm 2.8 \mu \mathrm{L}$ vs. 31.1 $\pm 2.4 \mu \mathrm{L}, P<0.05, t=2.63$; CO: $16246 \pm 1320 \mu \mathrm{L} / \mathrm{min}$ vs. 11624

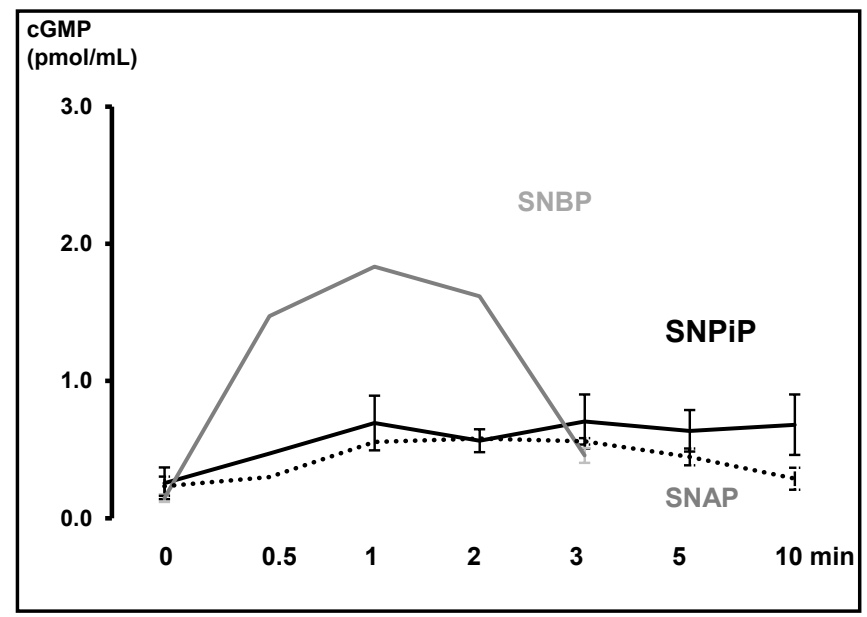

Fig. 5. Changes in cyclic guanidine monophosphate (cGMP) levels in SNPiP treatment compared to the levels in SNAP treatment. cGMP level of SNPiP-treated cells gradually increases without attaining a distinctive peak during the observation period, in contrast to the cGMP level in SNAP- and S-nitroso-Nbenzoyl-D-penicillamine (SNBP)-treated cells, where the peak levels are rapidly attained.

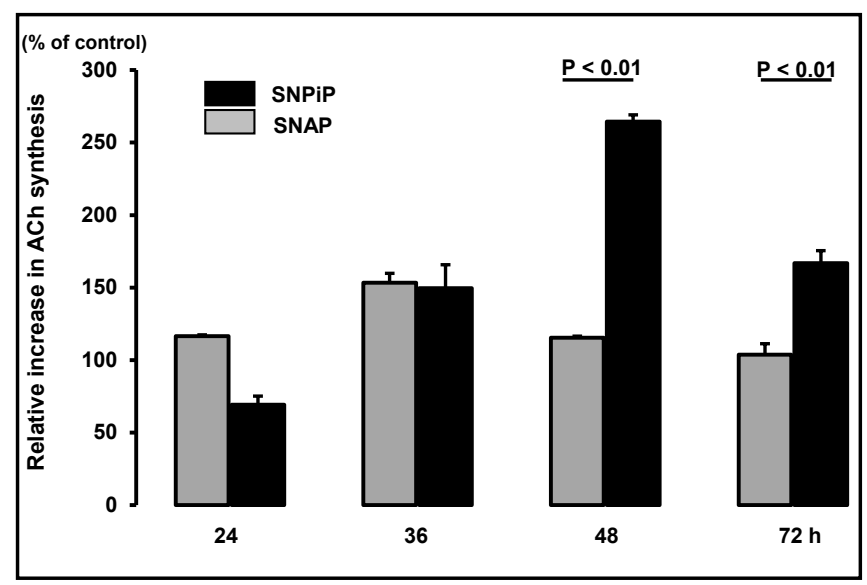

Fig. 6. SNPiP treatment accelerates ACh synthesis more than SNAP treatment within $72 \mathrm{~h}$. ACh synthesis increases to $153 \%$ with SNAP treatment, whereas its synthesis is much higher $(264 \%$ at $48 \mathrm{~h}, \mathrm{P}<0.01)$ and more prolonged $(167 \%$ at $72 \mathrm{~h}$, $\mathrm{P}<0.01$ ) with SNPiP treatment. $\pm 1172 \mu \mathrm{L} / \mathrm{min}, P<0.05, t=2.62$;

HR: $471 \pm 25$ vs. $453 \pm 25$ ), and the effects became gradually evident with more significant improvement in the cardiac function parameters at $72 \mathrm{~h}$.

A derivative of SNPiP without the nitroso group, PiP, loses the SNPiP-specific potential to upregulate the non-neuronal ACh synthesis system

To further understand which component of the chemical structure in SNPiP contributes to the induction of NNCCS, we synthesized PiP, which lacks the nitroso group of SNPiP (Fig. 1). In $1 \mu \mathrm{M}$ SNPiP-treated HEK293 cells, upregulated ChAT promoter activity $(155.5 \pm 11.1 \%)$ was observed at $72 \mathrm{~h}$. However, the ChAT activity of PiP-treated cells significantly decreased to $90.7 \pm 3.1 \%[P<0.01$ vs. control and PiP, $F(2,18)=23.6$, Fig 8$]$. Consistently, PiP did not 
upregulate the ChAT protein expression. This suggested that the nitroso group of SNPiP was responsible for ChAT induction.

Seventy two hours after PiP administration in mice, it did not increase SV [PiP: $23.7 \pm$ $0.4 \mu \mathrm{L}$ vs. SNPiP $31.5 \pm 2.0, \mathrm{~F}(2,26)=13.7, P<0.01]$, EDV [PiP: $31.6 \pm 0.8 \mu \mathrm{L}$ vs. SNPiP $38.2 \pm$ $3.0, \mathrm{~F}(2,26)=6.0, P<0.05]$, or CO [PiP: $9085.3 \pm 434.4 \mu \mathrm{L} / \mathrm{min}$ vs. SNPiP $12639.1 \pm 1076.9$, $\mathrm{F}(2,26)=8.2, P<0.01]$ to the levels observed with SNPiP-treatment. These results also indicate that the nitroso group is responsible for increasing the murine cardiac function via NNCCS upregulation.

\section{Discussion}

This is the first study to report a novel compound SNPiP, which plays a pivotal role in activating the non-neuronal cholinergic system (NNCS), and therefore, acts as an inducer of NNCS. This compound slowly upregulated the transcription and translation of ChAT in vitro, gradually increasing ACh synthesis. This involved a delayed peak of NO release and intracellular cGMP levels.

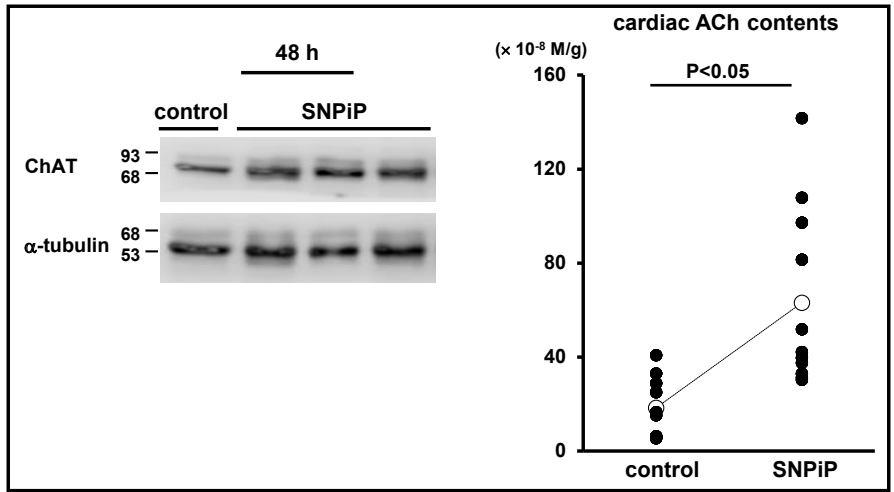

Fig. 7. SNPiP increases ACh synthesis in the heart through ChAT protein expression. Increased ChAT expression is observed 48 $\mathrm{h}$ after SNPiP-treatment in murine heart. This is followed by an increased ACh content in the heart $72 \mathrm{~h}$ after the treatment $(\mathrm{P}<0.05)$.

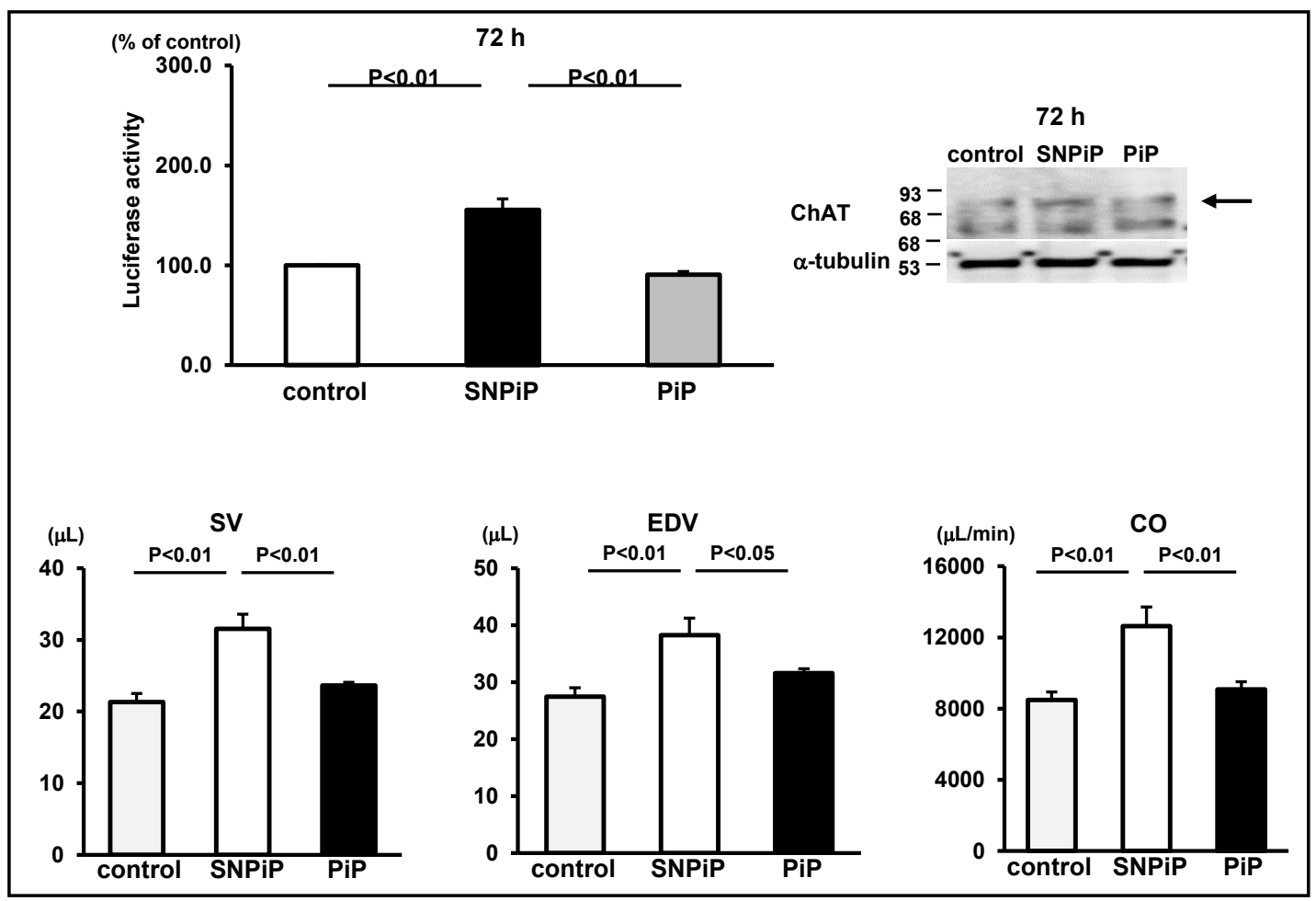

Fig. 8. N-pivaloyl-D-penicillamine (PiP) lacking the nitroso group of SNPiP does not present SNPiP characters. PiP upregulates neither the ChAT protein expression nor the luciferase activity, even $72 \mathrm{~h}$ after the treatment $(\mathrm{P}<0.01$ vs. SNPiP). The SV, EDV, and CO parameters, which are significantly upregulated by SNPiP treatment, are attenuated to control levels with $\mathrm{PiP}$ treatment $(\mathrm{P}<0.01$ or $\mathrm{P}<0.05$ vs. SNPiP). 
Thereafter, SNPiP was revealed to activate ACh synthesis in the murine heart in vivo. In contrast, SNPiP did not activate ACh synthesis in the skeletal muscle, which is believed to be responsible for non-neuronal ACh synthesis. SNPiP further increased the mice cardiac function with augmented SV, ESV, and CO. Therefore, it is proposed that SNPiP plays a role of an activator of NNCCS to increase cardiac function.

We have earlier reported that cardiomyocytes produce ACh using the NNCCS [1]. Since the establishment of this concept [9-14], we have revealed the physiological and pathophysiological roles of NNCCS to advocate for its integral effects in a general cardiac function, which includes accelerating cell-cell communication and angiogenesis [2, 3, 15], protecting cardiomyocytes from ROS and ischemic insults $[2,3]$, and modulating cardiac energy metabolism $[3,16]$.

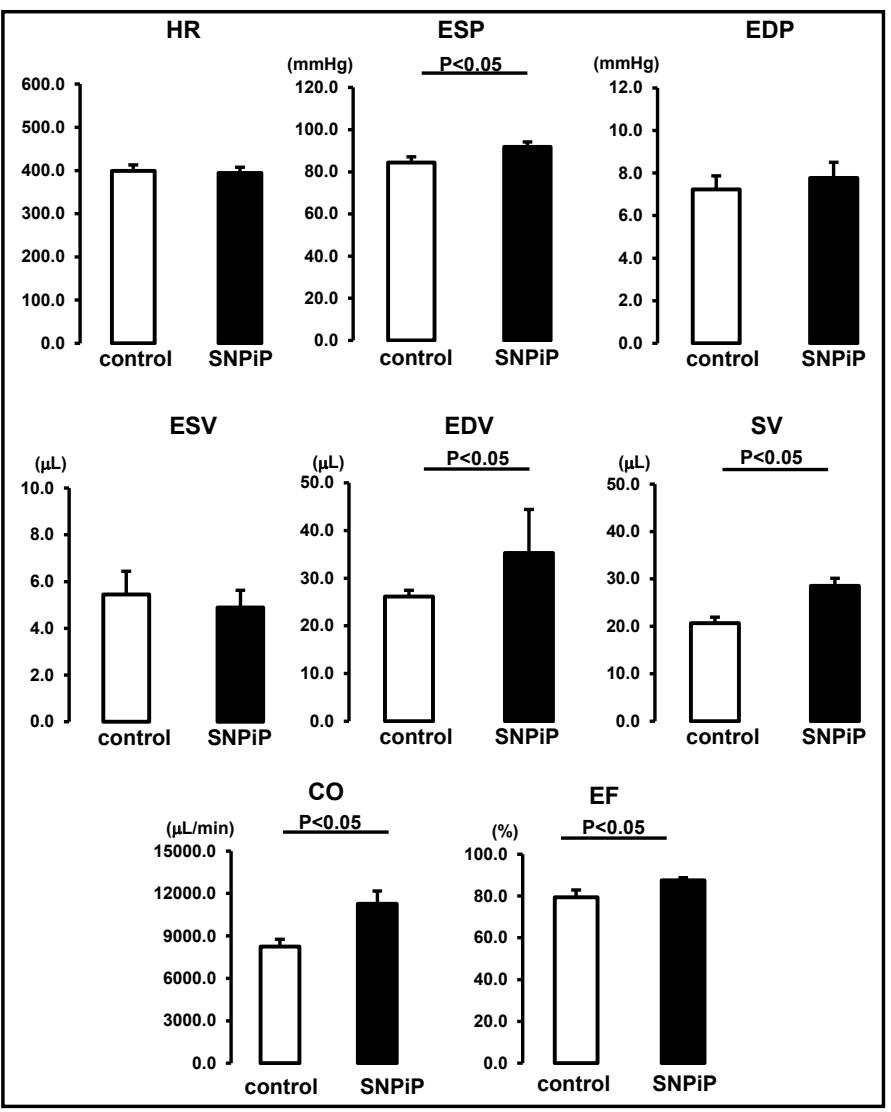

Fig. 9. SNPiP facilitates murine cardiac function. After $72 \mathrm{~h}$, the SNPiP-treated mice revealed increase in the EDV $(\mathrm{P}<0.05)$ and SV $(\mathrm{P}<0.05)$, leading to increases in $\mathrm{CO}(\mathrm{P}<0.05)$ and $\mathrm{EF}(\mathrm{P}<0.05)$, and consequently elevated ESP $(\mathrm{P}<0.05)$.

These pivotal roles of

NNCCS, therefore, have prompted us to further search for a modality to activate NNCCS using the pharmacological approach using donepezil $[1,15]$ and remote ischemic preconditioning $[2,5]$. In our previous study, we identified that donepezil, an anti-Alzheimer's disease drug, could upregulate NNCCS; intriguingly, donepezil also improved the impaired cardiac function caused by myocardial infarction in animal models due to its cholinergic activation effects. This is because it has been recognized as an acetylcholinesterase inhibitor that reduces HR, such as $\beta$-blockers [17-19]. Furthermore, it was previously reported that donepeziltreated Alzheimer's patients were less susceptible to cardiovascular diseases and survived longer [20-22]. Collectively, one of the mechanisms of donepezil may involve the restoration of downregulated cholinergic tonus in the autonomic nervous system in vivo $[21,22]$ in addition to functioning as an NNCCS inducer.

Till date, no other NNCCS inducer has been reported [1]. Therefore, the present study was performed to identify pharmacological agents that can induce NNCCS. Mouse with heart-specific ChAT gene overexpressing (ChAT tgm), an in vivo NNCCS activated model [3], had previously shown that increased cardiac NO was responsible for the characteristic phenotypes of ChAT tgm including anti-anxiety, anti-depression, anti-stress, and anticonvulsion [4]. These results further prompted us to consider NO donors as suitable candidates for NNCCS activation.

The current study shows that SNAP, despite being a short-acting vasodilator, increased the ChAT protein expression along with enhanced NO release $[23,24]$. NO donors-treated animal often exhibited decreased blood pressure $[8,25]$, although SNAP played another crucial role in HIF-1 $\alpha$ induction [26]. 


\section{Cellular Physiology Cell Physiol Biochem 2019;52;922-934

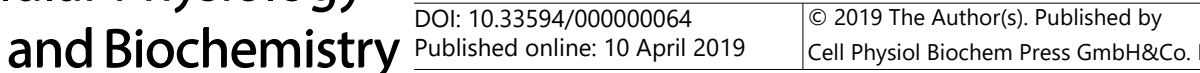

Specifically, SNPiP gradually activated the reporter activity and protein expression of ChAT to a lower extent for over $30 \mathrm{~h}$. Molecular mechanisms for the ChAT expression modes may contribute to distinctive cGMP regulation because SNPiP-treatment leads to gradual elevation of the cGMP levels. This was further confirmed by the delayed peak of NO release by SNPiP treatment. These different signal transduction patterns may cause a delayed initiation in ACh synthesis by SNPiP in vitro. SNPiP gradually upregulated the cardiac ACh content during the time course, and ACh synthesis was at its peak in the heart at $72 \mathrm{~h}$. This gradual induction of cardiac ACh synthesis was expected to be advantageous as it revealed mild hemodynamic effects (HR and BP) on the SNPiP-administered animal models.

As SNPiP has the advantage of upregulating ACh synthesis specifically in the heart, but not in the skeletal muscle, it is suggested to be an inducer of NNCCS when administered in vivo. The mechanisms underlying this specific upregulation was not completely investigated in this study. However, SNPiP and SNAP differed in their chemical composition by only one functional group, which was attached to the base structure of SNAP by an amide bond. Therefore, we hypothesized that this additional group in SNPiP may contribute to this specificity as well as the long-acting ACh producing potency.

SNPiP evidently increased the cardiac hemodynamic performance (EDV, SV and CO) with comparable HR. This result suggests that SNPiP may increase cardiac wall compliance and induce more efficient dilatation of the heart. In contrast, PiP, which lacks the nitroso group, did not increase the ChAT promoter activity and ChAT protein expression. Consistently, PiP did not possess SNPiP-specific hemodynamic advantages in the heart. As a result, SNPiP (and not PiP) further elevated $\mathrm{CO}$, which is associated with the increase in ESP, presumably via the law of Frank-Starling. If SNPiP rapidly decreased the vascular tone and dilated vasculature via the released NO, blood pressure should decrease with increasing HR. However, HR and BP were not significantly altered by SNPiP, rather, ESP was increased with elevated CO. These effects of SNPiP on the heart seem to be due to the slow induction of NNCCS, similar to the effects of observed in the cardiac function of ChAT tgm [3].

NO has been reported to have an LV relaxation-hastening effect to increase the diastolic $\mathrm{LV}$ distensibility and LV preload reserve, thereby upregulating the cardiac performance [27-29]. Therefore, SNPiP-induced improvement of diastolic function may contribute to upregulated ACh-induced NO causing cardiac function alteration. Therefore, taken together with the PiP-related results, NO derived from SNPiP may be crucial in upregulating NNCCS.

Another advantage of SNPiP-mediated cardiac modulation was that it did not increase HR, suggesting that SNPiP may not upregulate cardiac oxygen consumption. ACh and NO negatively regulates the cardiac oxygen consumption $[30,31]$. Therefore, these characteristics of SNPiP, in addition to the induction of NNCCS, may render it superior to other agents. As mentioned thus far, SNPiP with a potency to increase cardiac function exhibited a good contrast to sympathetic nerve-mediated adrenergic stimulation or inotropic agents, suggesting that SNPiP has a specific advantage.

In the present study, we explored a limited number of NO donor-based chemical compounds. Therefore, we do not exclude any possibility that more potent chemicals can induce NNCCS than SNPiP. However, the results of this study suggest that SNPiP is superior to other derivatives of SNAP. 


\section{Cellular Physiology Cell Physiol Biochem 2019;52;922-934 \\ \begin{tabular}{l|l} 
DOI: 10.33594/000000064 & (c) 2019 The Author(s). Published by
\end{tabular} \\ Published online: 10 April $2019 \quad$ Cell Physiol Biochem Press GmbH\&Co. KG \\ Oikawa et al.: S-Nitroso-N-Pivaloyl-D-Penicillamine Activates Cardiac ACh Synthesis}

\section{Conclusion}

SNPiP was observed to activate NNCCS to increase cardiac ACh synthesis thereby contributing to an increased cardiac performance, suggesting that SNPiP may be categorized into a novel group as an NNCCS inducer.

\section{Acknowledgements}

Kakinuma (Y. K.) conceived and designed the experiments. Oikawa (S. O.) and Kai (Y. K.) performed the experiments. Nakamura (S. N.) synthesized compounds. Mano (A. M.) contributed analysis tools. Y. K. and S. N. wrote and edited the manuscript.

We thank Cactus Communications Inc., Tokyo, Japan, for editing our manuscript. This manuscript has been critically checked for language by their native English speakers.

This study was partially supported by the Japan Society for the Promotion of Science Grants-in-Aid for Scientific Research (JSPS KAKENHI) (C) (Grant Number: 16K08560), and partly supported by Smoking Research Foundation.

The present animal study was approved by the ethical committee of Nippon Medical School (permission number 27-0003), and all animal experiments were performed in strict accordance with the recommendations set in the ARRIVE guidelines and carried out in accordance with National Institutes of Health guide for the care and use of laboratory animals.

\section{Disclosure Statement}

There is no conflict of interests to declare.

\section{References}

1 Kakinuma Y, Akiyama T, Sato T: Cholinoceptive and cholinergic properties of cardiomyocytes involving an amplification mechanism for vagal efferent effects in sparsely innervated ventricular myocardium. FEBS J 2009;276:5111-5125.

2 Kakinuma Y, Akiyama T, Okazaki K, Arikawa M, Noguchi T, Sato T: A non-neuronal cardiac cholinergic system plays a protective role in myocardium salvage during ischemic insults. PLoS One 2012;7:e50761.

3 Kakinuma Y, Tsuda M, Okazaki K, Akiyama T, Arikawa M, Noguchi T, Sato T: Heart-specific overexpression of choline acetyltransferase gene protects murine heart against ischemia through hypoxia-inducible factor$1 \alpha$-related defense mechanisms. J Am Heart Assoc 2013;2:e004887.

4 Oikawa S, Kai Y, Tsuda M, Ohata H, Mano A, Mizoguchi N, Sugama S, Nemoto T, Suzuki K, Kurabayashi A, Muramoto K, Kaneda M, Kakinuma Y: Non-neuronal cardiac cholinergic system influences CNS via the vagus nerve to acquire a stress-refractory propensity. Clin Sci (Lond) 2016;130:1913-1928.

5 Oikawa S, Mano A, Takahashi R, Kakinuma Y: Remote ischemic preconditioning with a specialized protocol activates the non-neuronal cardiac cholinergic system and increases ATP content in the heart. Int Immunopharmacol 2015;29:181-184.

6 Basalay MV, Davidson SM, Gourine AV, Yellon DM: Neural mechanisms in remote ischaemic conditioning in the heart and brain: mechanistic and translational aspects. Basic Res Cardiol 2018;113:25.

7 Kurabayashi A, Tanaka C, Matsumoto W, Naganuma S, Furihata M, Inoue K, Kakinuma Y: Murine remote preconditioning increases glucose uptake and suppresses gluconeogenesis in hepatocytes via a brain-liver neurocircuit, leading to counteracting glucose intolerance. Diabetes Res Clin Pract 2018;139:288-299.

8 Styś T, Styś A, Paczwa P, Szczepańska-Sadowska E, Lipkowski AW: Decreased hypotensive responsiveness to nitric oxide donor S-nitroso N-acetyl-DL-penicillamine (SNAP) in spontaneously hypertensive (SHR) rats. J Physiol Pharmacol 1998;49:37-49. 


\section{Cellular Physiology Cell Physiol Biochem 2019;52;922-934 \begin{tabular}{l|l|l}
\hline DOI: 10.33594/000000064 & (c) 2019 The Author(s). Published by
\end{tabular} and Biochemistry Published online: 10 April $2019 \quad$ Cell Physiol Biochem Press GmbH\&Co. KG

9 Rana OR, Schauerte P, Kluttig R, Schröder JW, Koenen RR, Weber C, Nolte KW, Weis J, Hoffmann R, Marx $\mathrm{N}$, Saygili E: Acetylcholine as an age-dependent non-neuronal source in the heart. Auton Neurosci 2010;156:82-89.

- 10 Rocha-Resende C, Roy A, Resende R, Ladeira MS, Lara A, de Morais Gomes ER, Prado VF, Gros R, Guatimosim C, Prado MA, Guatimosim S: Non-neuronal cholinergic machinery present in cardiomyocytes offsets hypertrophic signals. J Mol Cell Cardiol 2012;53:206-216.

- 11 Roy A, Fields WC, Rocha-Resende C, Resende RR, Guatimosim S, Prado VF, Gros R, Prado MA: Cardiomyocyte-secreted acetylcholine is required for maintenance of homeostasis in the heart. FASEB J 2013;27:5072-5082.

- 12 Gavioli M, Lara A, Almeida PW, Lima AM, Damasceno DD, Rocha-Resende C, Ladeira M, Resende RR, Martinelli PM, Melo MB, Brum PC, Fontes MA, Souza Santos RA, Prado MA, Guatimosim S: Cholinergic signaling exerts protective effects in models of sympathetic hyperactivity-induced cardiac dysfunction. PLoS One 2014;9:e100179.

13 Durand MT, Becari C, Tezini GC, Fazan R Jr, Oliveira M, Guatimosim S, Prado VF, Prado MA, Salgado HC: Autonomic cardiocirculatory control in mice with reduced expression of the vesicular acetylcholine transporter. Am J Physiol Heart Circ Physiol 2015;309:H655-662.

- 14 Roy A, Dakroub M, Tezini GC, Liu Y, Guatimosim S, Feng Q Salgado HC, Prado VF, Prado MA, Gros R: Cardiac acetylcholine inhibits ventricular remodeling and dysfunction under pathologic conditions. FASEB J 2016;30:688-701.

- 15 Kakinuma Y, Furihata M, Akiyama T, Arikawa M, Handa T, Katare RG, Sato T: Donepezil, an acetylcholinesterase inhibitor against Alzheimer's dementia, promotes angiogenesis in an ischemic hindlimb model. J Mol Cell Cardiol 2010;48:680-693.

- 16 Oikawa S, Iketani M, Kakinuma Y: A non-neuronal cholinergic system regulates cellular ATP levels to maintain cell viability. Cell Physiol Biochem 2014;34:781-789.

- 17 Handa T, Katare RG, Kakinuma Y, Arikawa M, Ando M, Sasaguri S, Yamasaki F, Sato T: Anti-Alzheimer's drug, donepezil, markedly improves long-term survival after chronic heart failure in mice. J Card Fail 2009;15:805-811.

18 Arikawa M, Kakinuma Y, Handa T, Yamasaki F, Sato T: Donepezil, anti-Alzheimer's disease drug, prevents cardiac rupture during acute phase of myocardial infarction in mice. PLoS One 2011;6:e20629.

19 Okazaki Y, Zheng C, Li M, Sugimachi M: Effect of the cholinesterase inhibitor donepezil on cardiac remodeling and autonomic balance in rats with heart failure. J Physiol Sci 2010;60:67-74.

20 Sato K, Urbano R, Yu C, Yamasaki F, Sato T, Jordan J, Robertson D, Diedrich A: The effect of donepezil treatment on cardiovascular mortality. Clin Pharmacol Ther 2010;88:335-338.

- 21 Nordström P, Religa D, Wimo A, Winblad B, Eriksdotter M: The use of cholinesterase inhibitors and the risk of myocardial infarction and death: a nationwide cohort study in subjects with Alzheimer's disease. Eur Heart J 2013;34:2585-2591.

- 22 Wu PH, Lin YT, Hsu PC, Yang YH, Lin TH, Huang CT: Impact of acetylcholinesterase inhibitors on the occurrence of acute coronary syndrome in patients with dementia. Sci Rep 2015;5:15451.

23 Megson IL, Greig IR, Gray GA, Webb DJ, Butler AR: Prolonged effect of a novel S-nitrosated glyco-amino acid in endothelium-denuded rat femoral arteries: potential as a slow release nitric oxide donor drug. $\mathrm{Br} \mathrm{J}$ Pharmacol 1997;122:1617-1624.

24 Eduardo da Silva-Santos J, Assreuy J: Long-lasting changes of rat blood pressure to vasoconstrictors and vasodilators induced by nitric oxide donor infusion: involvement of potassium channels. J Pharmacol Exp Ther 1999;290:380-387.

25 Schyvens CG, Cowden WB, Zhang Y, McKenzie KU, Whitworth JA: Hemodynamic effects of the nitric oxide donor DETA/NO in mice. Clin Exp Hypertens 2004;26:525-535.

26 Kuwabara M, Kakinuma Y, Ando M, Katare RG, Yamasaki F, Doi Y, Sato T: Nitric oxide stimulates vascular endothelial growth factor production in cardiomyocytes involved in angiogenesis. J Physiol Sci 2006;56:95101.

- 27 Paulus WJ, Bronzwaer JG: Myocardial contractile effects of nitric oxide. Heart Fail Rev 2002;7:371-383.

- 28 Brunner F, Maier R, Andrew P, Wölkart G, Zechner R, Mayer B: Attenuation of myocardial ischemia/ reperfusion injury in mice with myocyte-specific overexpression of endothelial nitric oxide synthase. Cardiovasc Res 2003;57:55-62. 
Cellular Physiology Cell Physiol Biochem 2019;52;922-934

\begin{tabular}{ll|l} 
and Biochemistry & $\begin{array}{l}\text { DOl: } 10.33594 / 000000064 \\
\text { Published online: } 10 \text { April } 2019\end{array}$ & $\begin{array}{l}\text { C) } 2019 \text { The Author(s). Published by } \\
\text { Cell Physiol Biochem Press GmbH\&Co. KG }\end{array}$ \\
\cline { 2 - 3 } & Oikawa et al.: S-Nitroso-N-Pivaloyl-D-Penicillamine Activates Cardiac ACh Synthesis
\end{tabular}

29 Wilson RM, De Silva DS, Sato K, Izumiya Y, Sam F: Effects of fixed-dose isosorbide dinitrate/hydralazine on diastolic function and exercise capacity in hypertension-induced diastolic heart failure. Hypertension 2009;54:583-590.

- 30 Walsh EK, Huang H, Wang Z, Williams J, de Crom R, van Haperen R, Thompson CI, Lefer DJ, Hintze TH: Control of myocardial oxygen consumption in transgenic mice overexpressing vascular eNOS. Am J Physiol Heart Circ Physiol 2004;287:H2115-2121.

- 31 Kinugawa S, Huang H, Wang Z, Kaminski PM, Wolin MS, Hintze TH: A defect of neuronal nitric oxide synthase increases xanthine oxidase-derived superoxide anion and attenuates the control of myocardial oxygen consumption by nitric oxide derived from endothelial nitric oxide synthase. Circ Res 2005;96:355362. 